\title{
SIMULAÇÃO DAS PERDAS DE ÁGUA POR EVAPORAÇÃO E ARRASTE, NO ASPERSOR NY-7 (4,6 mm x 4,0 mm), EM SISTEMAS DE ASPERSÃO CONVENCIONAL
}

\section{SAMUEL BESKOW ${ }^{1}$, ALBERTO COLOMBO ${ }^{2}$, MOISÉS S. RIBEIRO ${ }^{3}$, LIAMARA S. FERREIRA ${ }^{4}$, RAITANNI ROSSI ${ }^{4}$}

\begin{abstract}
RESUMO: As perdas de água por evaporação e arraste em sistemas de irrigação por aspersão podem assumir valores consideráveis, reduzindo a eficiência do sistema. Os objetivos do presente trabalho foram avaliar a capacidade preditiva de cinco modelos empíricos para estimar perdas de água por evaporação e arraste em aspersores modelo NY-7 (bocais de 4,6 mm x 4,0 mm), trabalhando sob diferentes condições operacionais e ambientais, e ajustar modelos específicos para o aspersor NY-7. Comparando os resultados medidos em ensaios de campo, com os resultados simulados, foi possível concluir que os cinco modelos empíricos considerados apresentaram pouca ou nenhuma adequação, tanto para os ensaios com um único aspersor (quadrado do erro-médio de 5,$27 ; 20,70 ; 5,07 ; 6,95$ e 7,06\% para os modelos empíricos $1 ; 2 ; 3 ; 4$ e 5 , respectivamente) quanto para os ensaios com linhas laterais contendo aspersores (quadrado do erro-médio de 7,41; 24,43; 6,$72 ; 3,16$ e 2,9\% para os modelos empíricos $1 ; 2 ; 3 ; 4$ e 5, respectivamente). Comparados aos cinco modelos empíricos considerados, os novos modelos ajustados apresentaram menores erros, indicando que a aplicação de modelos empíricos deve ser limitada às condições de operação (diâmetro de bocal, pressão de operação, etc.) similares àquelas em que os modelos foram desenvolvidos.
\end{abstract}

PALAVRAS-CHAVE: modelos empíricos, eficiência de irrigação, modelagem matemática.

\section{SIMULATION OF EVAPORATION AND WIND DRIFT LOSSES, IN THE NY-7 SPRINKLER (4.6 mm x $4.0 \mathrm{~mm})$, IN STATIONARY SPRINLER IRRIGATION SYSTEMS}

\begin{abstract}
Evaporation and wind drift losses during sprinkler irrigation may reach significant values, cutting system efficiency down. The present work aims: (a) to evaluate the ability of five empirical models in predicting losses of a NY-7 model sprinkler (nozzle of $4.6 \mathrm{~mm} \times 4.0 \mathrm{~mm}$ ), working under different operational and climatic conditions; and (b) to adjust specific models to the NY-7 sprinkler. By comparing measured values - obtained on field trials - with simulated ones, it was possible to conclude that, in general, the five considered empirical models presented little or no adjustment for the single-sprinkler outdoor tests (root mean square error of $5.27 ; 20.70 ; 5.07 ; 6.95$ and $7.06 \%$ for empirical models $1 ; 2 ; 3 ; 4$ and 5 , respectively) as well as for the block irrigation outdoor tests (root mean square error of $7.41 ; 24.43 ; 6.72 ; 3.16$ and $2.90 \%$ for empirical models 1 ; $2 ; 3 ; 4$ and 5, respectively). When compared to the five considered empirical models, the new adjusted models showed lower errors, indicating that the application of empirical models must be limitated to working conditions (nozzle size, operational pressure, etc.) similar to the ones in which they were developed.
\end{abstract}

KEYWORDS: empirical models, irrigation efficiency, mathematical modeling.

\footnotetext{
${ }^{1}$ Eng ${ }^{\circ}$ Agrícola, Doutorando em Engenharia Agrícola, Departamento de Engenharia, UFLA, Lavras - MG, Fone: (0XX35) 3822.6604, samuelbeskow@terra.com.br

${ }^{2}$ Eng ${ }^{\circ}$ Agrônomo, Prof. Adjunto, Departamento de Engenharia, UFLA, Lavras - MG.

${ }^{3}$ Eng ${ }^{0}$ Agrônomo, Doutorando em Engenharia Agrícola, Departamento de Engenharia, UFLA, Lavras - MG.

${ }^{4}$ Eng $^{\mathrm{O}}$ Agrícola, UFPel, Pelotas - RS.

Recebido pelo Conselho Editorial em: 12-7-2007
}

Aprovado pelo Conselho Editorial em: 10-6-2008 


\section{INTRODUÇÃO}

A busca por novos conceitos e estratégias para otimizar a eficiência de utilização da água tem sido objeto de estudo de diversos pesquisadores. De acordo com KELLER \& BLIESNER (1990), muita preocupação tem sido relatada à avaliação de sistemas de irrigação, porém perdas de água também reduzem a eficiência do sistema e, freqüentemente, projetistas assumem que os sistemas serão perfeitamente manejados, e as perdas serão mínimas, mas isso raramente acontece. Os mesmos autores dizem que a irrigação em excesso seja, talvez, a maior causa de perdas em um sistema de irrigação, mas que outras fontes de perdas associadas à irrigação por aspersão são: evaporação das gotas e superfícies úmidas do solo, arraste pelo vento, vazamentos e sistemas de drenagem.

SMAJSTRLA \& ZAZUETA (2003) relatam que a perda de água por evaporação é aquela parcela de água que evapora das gotas pulverizadas pelo ar, e a perda por arraste diz respeito à água carregada pelo vento para fora da área irrigada. Os principais fatores que interferem nos valores de perdas de água por evaporação e arraste, na irrigação por aspersão, são, segundo TARJUELO (1999), umidade relativa do ar, temperatura do ar e da água, altura do aspersor, tamanho de gotas e velocidade do vento.

A fim de determinar as perdas de água por evaporação e arraste na irrigação por aspersão, podem ser utilizados métodos de campo, analíticos e de laboratório (KOHL et al., 1987). Essas perdas obtidas experimentalmente no campo variam entre $2 \%$ e $40 \%$, com muitos valores dentro do intervalo de 10\%-20\% (KINCAID, 1996; KINCAID et al., 1996; KOHL et al., 1987; YAZAR, 1984), enquanto os valores obtidos de forma analítica ou por ensaios realizados em laboratório apresentam valores de $0,5 \%$ a $2 \%$ (KOHL et al., 1987).

Os modelos matemáticos servem como alternativa de superar muitos erros decorrentes de medições na avaliação de perdas de água na aspersão durante a irrigação (THOMPSON et al., 1993). PLAYÁN et al. (2005) fizeram uma revisão acerca de modelos existentes na bibliografia para a estimativa das perdas de água por evaporação e arraste na irrigação por aspersão, destacando as variáveis ambientais e operacionais consideradas em cada modelo proposto.

FACI et al. (2001), ao realizarem testes de campo com aspersores do tipo spray e compararem os valores obtidos com os simulados pelos modelos de KELLER \& BLIESNER (1990), MONTERO et al. (1997) e TRIMMER (1987), chegaram à conclusão de que os três modelos utilizados subestimaram os valores de perdas obtidos a partir de testes de campo.

Em experimento realizado em Zaragoza (Espanha), PLAYÁN et al. (2005) compararam os resultados de perdas de água obtidos por meio de avaliações de campo com os resultados gerados por oito modelos disponíveis na bibliografia. Segundo os mesmos autores, o ajuste das equações com os dados de campo foi desanimador, sendo que tais equações tendem a gerar melhores resultados (ou seja, perdas menores) quando comparados com o que realmente acontece no sistema de irrigação.

Este trabalho teve os objetivos de simular as perdas de água por evaporação e arraste em um sistema de aspersão convencional com aspersores NY-7 (bocais de 4,6 mm x 4,0 mm e ângulo de inclinação de $7^{\circ}$ ) sob diferentes condições operacionais e ambientais, segundo cinco diferentes modelos empíricos disponíveis na bibliografia, e comparar com os valores obtidos por meio de testes de campo; avaliar a capacidade e as limitações de cada modelo empírico utilizado, e ajustar modelos específicos para a configuração do aspersor em questão.

\section{MATERIAL E MÉTODOS}

A parte experimental deste trabalho foi conduzida durante os meses de março e abril de 2006, em área localizada na Universidade Federal de Lavras (Lavras - MG), utilizando um sistema de aspersão convencional com um único aspersor operando, e também com vários aspersores operando simultaneamente (BESKOW, 2006). 
Para a realização dos testes, utilizaram-se aspersores Agropolo modelo NY-7, com ângulo de inclinação de $7^{\circ}$ e bocais de 4,6 mm x 4,0 mm, operando nas pressões $245 \mathrm{kPa}, 294 \mathrm{kPa}$ e $343 \mathrm{kPa}$.

Durante os ensaios de campo, foi necessário monitorar algumas variáveis meteorológicas de interesse, e, para isso, fez-se uso de estação automática, modelo Vantage Pro2, localizada acerca de $20 \mathrm{~m}$ da área de irrigação. Essa estação registrava as seguintes variáveis meteorológicas: (a) temperatura e umidade relativa do ar a $2 \mathrm{~m}$ acima do nível do solo, e (b) velocidade e direção do vento a $2 \mathrm{~m}$ de altura acima do nível do solo. Os dados dessas variáveis foram registrados de forma manual, com freqüência de cinco minutos.

Dois tipos de ensaios foram realizados: (a) com um único aspersor operando, e (b) com vários aspersores operando simultaneamente.

No primeiro tipo de teste (Figura 1), o aspersor foi locado no centro de uma malha de coletores composta de 144 coletores, formando quadrados de $2 \mathrm{~m}$ de lado. O aspersor estava à altura de $1,25 \mathrm{~m}$, propiciando diferença de altura de $0,75 \mathrm{~m}$ do bocal do aspersor em relação à abertura dos coletores. Para o armazenamento de água proveniente dos aspersores durante os testes, foram utilizadas latas de óleo (de cozinha) como coletores, pintadas na cor branca. O diâmetro interno do coletor foi de $0,0835 \mathrm{~m}$ e altura igual a $0,190 \mathrm{~m}$.

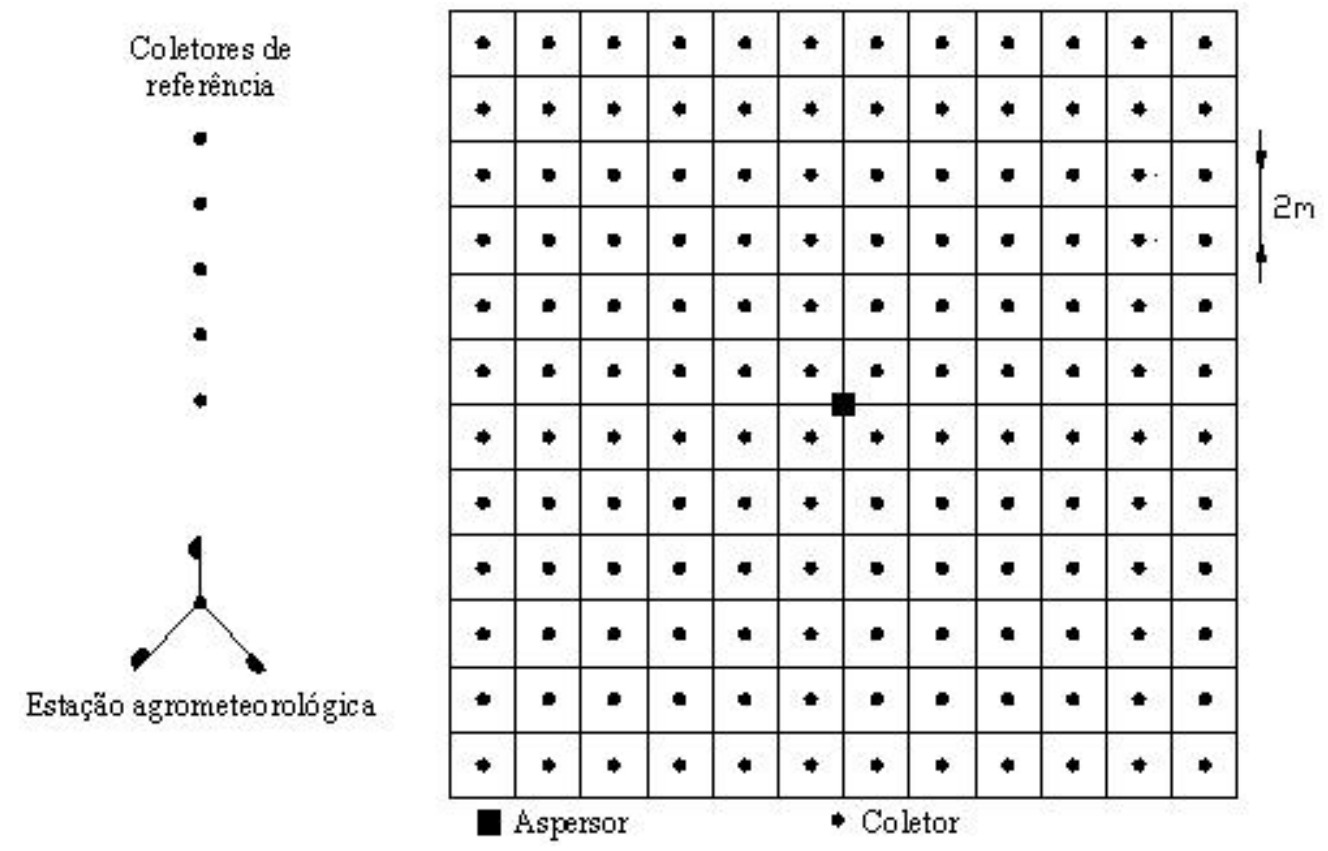

FIGURA 1. Esquema da disposição dos coletores, do aspersor e da estação agrometeorológica para o teste com um único aspersor. Schematic representation of the experimental set-up for single-sprinkler trials, including catch cans, sprinkler and weather station.

A fim de monitorar a pressão na entrada do bocal do aspersor, durante todo o teste de irrigação, utilizou-se de manômetro de Bourdon, graduado de $0 \mathrm{kPa}$ a $785 \mathrm{kPa}$, com intervalos de $5 \mathrm{kPa}$ (aproximadamente). Com o objetivo de aumentar ou diminuir a pressão de operação do aspersor, foi instalada uma válvula de gaveta na derivação da linha lateral para o tubo de subida do aspersor.

A duração de cada avaliação realizada, para esse tipo de teste (com um único aspersor), foi de 60 minutos. O volume armazenado dentro de cada coletor foi medido por proveta graduada, logo após o término da irrigação, e, em seguida, esses valores foram registrados em planilha apropriada. O tempo de medição foi entre 20 e 30 minutos. Durante o período de irrigação, a cada cinco minutos, foram registrados, por meio de estação agrometeorológica automática, os dados de temperatura do ar, umidade relativa do ar e velocidade e direção do vento. 
A fim de tentar estimar a evaporação nos coletores durante a leitura dos volumes, foram dispostos cinco coletores como referência, posicionados $20 \mathrm{~m}$ distante da área de testes. Esses coletores continham volumes conhecidos (semelhantes aos esperados de coletar). Os volumes foram medidos novamente após o término da leitura dos volumes contidos nos coletores. Em cada coletor, o volume de água coletado foi corrigido a partir da evaporação ocorrida durante o processo de leitura. A leitura de cada coletor foi aumentada em valor igual à metade da evaporação ocorrida durante o processo de leitura dos dados, estimada por meio dos coletores de referência, conforme recomenda TARJUELO et al. (2000).

Já no segundo tipo de ensaio (Figura 2), os aspersores foram dispostos em duas linhas laterais, com dois aspersores por linha lateral, no espaçamento de $12 \mathrm{~m} \mathrm{x} 12 \mathrm{~m}$. Os coletores foram dispostos de maneira a formarem quadrados de $2 \mathrm{~m}$ de lado. Cada teste tinha a duração de 50 minutos, e o processo de leitura dos volumes de água levava em torno de 10 minutos.

Para controlar a pressão no sistema durante o período de irrigação, foram utilizadas duas tomadas de pressão. Uma estava localizada entre uma válvula de gaveta e o primeiro aspersor, e a outra estava localizada no tubo de subida do último aspersor.

A operação, a obtenção dos dados meteorológicos e a correção da evaporação dos coletores durante a leitura dos dados foram realizadas da mesma maneira do teste com um único aspersor.

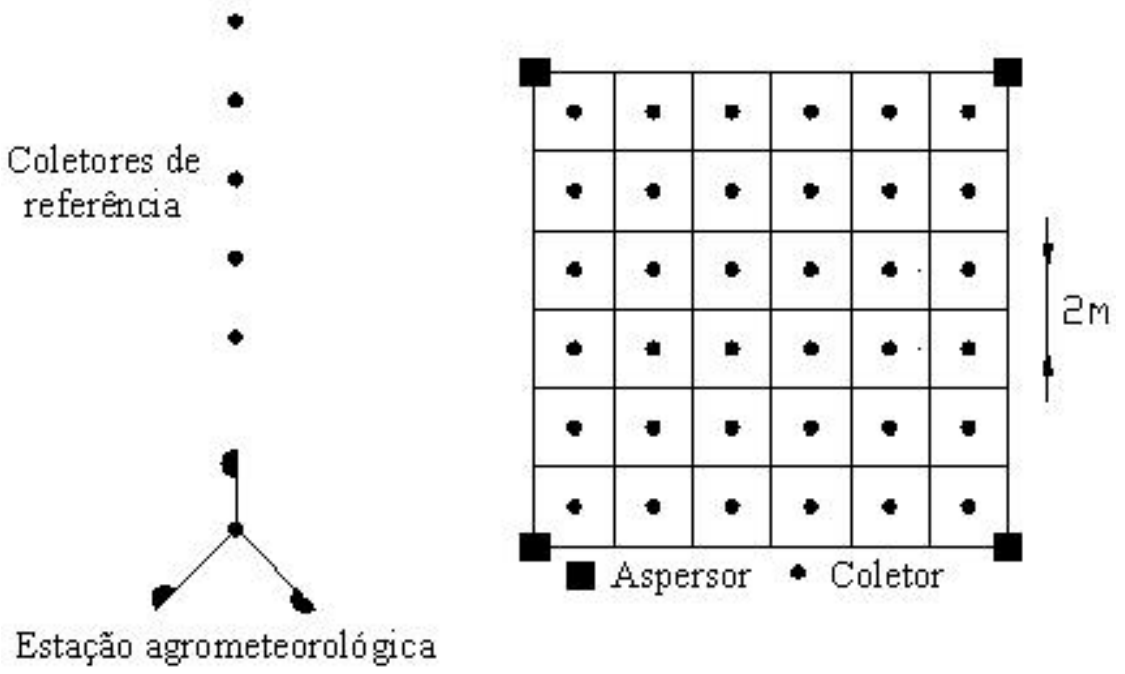

FIGURA 2. Esquema da disposição dos coletores, dos aspersores e da estação agrometeorológica para o teste com linhas laterais de aspersores. Schematic representation of the experimental set-up for block irrigation trials, including catch cans, sprinklers and weather station.

As perdas observadas por evaporação e arraste (EA) foram determinadas para cada avaliação como sendo igual à diferença entre o volume total aplicado pelo aspersor durante o período de irrigação e o volume total registrado nos coletores [eq.(1)]. As perdas por evaporação da água contida dentro dos coletores, durante o tempo de medição, foram consideradas e, portanto, acrescentadas a cada volume registrado nos coletores. Para os testes com um único aspersor, depois de corrigido o volume, foi realizada a sobreposição para o espaçamento de $12 \mathrm{~m}$ x $12 \mathrm{~m}$, de cada malha de dados, com o intuito de possibilitar o cálculo das perdas totais de água. Para os testes com laterais de aspersores, não foi necessário realizar o processo de sobreposição.

$$
\mathrm{EA}=\frac{\mathrm{Q}_{\mathrm{a}} \mathrm{t}-4 \sum_{\mathrm{i}=1}^{\mathrm{i}=36} \mathrm{Z}_{\mathrm{i}}}{\mathrm{Q}_{\mathrm{a}} \mathrm{t}} 100
$$

em que,

$\mathrm{Q}_{\mathrm{a}}$ - vazão do aspersor, $\mathrm{m}^{3} \mathrm{~h}^{-1}$;

$\mathrm{t}$ - duração da irrigação, $\mathrm{h}, \mathrm{e}$

$\mathrm{Z}_{\mathrm{i}}$ - lâmina armazenada em cada coletor, $\mathrm{m}$. 
O número 4 na eq.(1) significa que cada coletor representa uma área de $4 \mathrm{~m}^{2}$.

A vazão do aspersor, para cada pressão de serviço, foi obtida pelo processo direto (método gravimétrico), fixando-se um dado tempo no cronômetro e pesando-se o volume de água obtido nesse intervalo, em três repetições.

A fim de proceder à simulação das perdas de água por evaporação e arraste, para condições climáticas e operacionais idênticas às condições dos ensaios, foram utilizados os modelos de (1) PLAYÁN et al. (2005), (2) SEGINER et al. (1991), (3) TARJUELO et al. (2000), (4) TRIMMER (1987), e (5) YAZAR (1984), representados, respectivamente, pelas eq.(2); (3); (4); (5) e (6).

$$
\begin{aligned}
& \mathrm{EA}=20,3+0,214 \mathrm{U}^{2}-2,29 \times 10^{-3} \mathrm{UR}^{2} \\
& \mathrm{EA}=3,22 \exp ^{0,075 \mathrm{U}}\left(\mathrm{T}-\mathrm{T}_{\mathrm{w}}\right)^{0,69} \\
& \mathrm{EA}=0,007 \mathrm{P}_{\mathrm{S}}+7,38 \Delta \mathrm{e}^{0,5}+0,844 \mathrm{U} \\
& \mathrm{EA}=\left(1,98 \mathrm{D}^{-0,72}+0,22 \Delta \mathrm{e}^{0,63}+3,6 \times 10^{-4} \mathrm{P}_{\mathrm{S}}^{1,16}+0,14 \mathrm{U}^{0,7}\right)^{4,2} \\
& \mathrm{EA}=0,003 \exp ^{(0,20 \mathrm{U})}(10 \Delta \mathrm{e})^{0,59} \mathrm{~T}^{0,23} \mathrm{P}_{\mathrm{S}}^{0,76}+0,27 \mathrm{U}^{2,15}
\end{aligned}
$$

em que,

EA - perda de água por evaporação e arraste, \%;

D - diâmetro do bocal, mm;

$\Delta \mathrm{e}$ - déficit de pressão de vapor, $\mathrm{kPa}$;

$\mathrm{P}_{\mathrm{S}}$ - pressão de serviço do aspersor, $\mathrm{kPa}$;

$\mathrm{U}$ - velocidade média do vento, $\mathrm{m} \mathrm{s}^{-1}$;

$\mathrm{T}$ - temperatura do ar, ${ }^{\circ} \mathrm{C}$;

$\mathrm{T}_{\mathrm{w}}$ - temperatura de bulbo úmido, ${ }^{\circ} \mathrm{C}$, e

UR - umidade relativa do ar, \%.

Para o cálculo do déficit de pressão de vapor $(\Delta \mathrm{e})$, utilizou-se da eq.(7), recomendada por TRIMMER (1987).

$$
\Delta \mathrm{e}=0,61 \exp ^{\left(\frac{17,27 \mathrm{~T}}{\mathrm{~T}+237,3}\right)}\left(1-\frac{\mathrm{UR}}{100}\right)
$$

SEGINER et al. (1991) utilizaram a depressão de bulbo úmido $\left(\mathrm{T}-\mathrm{T}_{\mathrm{w}}\right)$ em vez do déficit de pressão de vapor. Para calcular a depressão de bulbo úmido, os mesmos autores recomendam a utilização da eq.(8):

$$
\Delta \mathrm{e}=0,00066\left(1+0,00115 \mathrm{~T}_{\mathrm{w}}\right)\left(\mathrm{T}-\mathrm{T}_{\mathrm{w}}\right) \mathrm{P}_{\mathrm{atm}}
$$

em que,

$\mathrm{P}_{\mathrm{atm}}$ - pressão atmosférica, $\mathrm{kPa}$.

Com o intuito de comparar os valores estimados pelos diferentes modelos com os valores observados, foram utilizados os seguintes indicadores estatísticos, conforme recomendado por CAMARGO \& SENTELHAS (1997): coeficiente de correlação (r); índice de Willmott (d), e índice de confiança ou desempenho (c). Para avaliar o desempenho dos modelos de simulação quanto aos valores de perdas de água por evaporação e arraste, seguiu-se o recomendado pelos mesmos autores: Ótimo ( $\mathrm{c}>0,85)$; Muito Bom $(0,76 \leq \mathrm{c} \leq 0,85)$; Bom $(0,66 \leq \mathrm{c} \leq 0,75)$; Mediano $(0,61 \leq \mathrm{c} \leq 0,65)$; Sofrível $(0,51 \leq \mathrm{c} \leq 0,60)$; Mau $(0,41 \leq \mathrm{c} \leq 0,50)$, e Péssimo $(\mathrm{c} \leq 0,40)$. 


\section{RESULTADOS E DISCUSSÃO}

A partir dos testes de campo, foi possível quantificar as perdas de água ocorridas por evaporação e arraste e, posteriormente, proceder à simulação das perdas de água sob as condições evidenciadas durante os testes, de acordo com os modelos de PLAYÁN et al. (2005), SEGINER et al. (1991), TARJUELO et al. (2000), TRIMMER (1987) e YAZAR (1984), a fim de averiguar a capacidade de tais modelos empíricos em prever valores de perdas de água por evaporação e arraste (Figuras 3 e 4).

O modelo de PLAYÁN et al. (2005), aplicado aos testes com um único aspersor (Figura 3a), mostrou desempenho "c" igual a 0,19 (Péssimo) e, quando se comparou com os resultados obtidos em ensaios com linhas laterais de aspersores (Figura 3b), foi possível constatar que o mesmo modelo apresentou tendência de superestimar os valores de perdas de água por evaporação e arraste, apresentando desempenho "Péssimo" $(\mathrm{c}=0,26)$. A diferença entre os resultados obtidos por simulação com esse modelo e os dados de campo pode ser justificada pelo tamanho de bocal utilizado para a geração do modelo, que, nesse caso, foram aspersores equipados com bocais de 4,4 mm x 2,4 mm (menores que os usados neste trabalho), acarretando, segundo SMAJSTRLA \& ZAZUETA (2003), maior pulverização das gotas e, conseqüentemente, apresentando maior área por unidade de massa (conseqüência na perda por evaporação) e maior suscetibilidade ao vento (reflete na perda por arraste pelo vento).

O modelo de SEGINER et al. (1991) gerou resultados de perdas excessivamente superiores àquelas obtidas tanto em testes com um único aspersor como também em testes com linhas laterais de aspersores (Figuras 3c e 3d), tendo desempenho "Péssimo" para os dois tipos de testes ("c" igual a 0,09 e 0,17, respectivamente). Esse modelo foi obtido a partir de testes com bocais de 3,5 mm, bastante inferior ao usado neste trabalho, acarretando valores de perdas de água por evaporação e arraste superiores, conforme justificado anteriormente. Outro aspecto relevante desse modelo é que ele foi desenvolvido por meio de testes de campo com um único aspersor, e sabe-se que, nesse tipo de teste, as perdas de água por evaporação e arraste são superiores, comparadas aos ensaios com linhas laterais de aspersores, visto que, no segundo tipo de ensaio, vários aspersores operam simultaneamente, propiciando microclima diferente daquele gerado nos ensaios com um único aspersor (ORTEGA et al., 2000).

Analisando as Figuras 3(e) e 3(f), é possível constatar que o modelo de TARJUELO et al. (2000) mostrou desempenho "Péssimo" ("c" igual a 0,17 e 0,33 , respectivamente) tanto para os testes com um único aspersor como também para os testes com linhas laterais de aspersores. No desenvolvimento de tal modelo, os autores fizeram uso de testes com linhas laterais de aspersores (equipados com bocais de $4,4 \mathrm{~mm}$ x $2,4 \mathrm{~mm}$ ), bocais esses menores que os usados no presente trabalho, acarretando perdas de água maiores, conforme justificativa já comentada anteriormente. PLAYÁN et al. (2005), ao realizarem testes de campo a fim de estimar perdas de água por evaporação e arraste, usaram o modelo de TARJUELO et al. (2000) para simular os valores de perdas, não encontrando ajuste satisfatório entre o modelo e os dados obtidos por meio de ensaios de campo, mesmo usando aspersores idênticos (marca e tamanho de bocal) aos usados para gerar tal modelo.

Quando se utilizou o modelo de TRIMMER (1987), pôde-se constatar que ele apresentou melhor concordância quando foi utilizado para os ensaios de campo com linhas laterais de aspersores (Figura 4b), mas, mesmo assim, apresentou índice de desempenho classificado como "Mau" (c = 0,46), segundo CAMARGO \& SENTELHAS (1997). TRIMMER (1987) gerou uma equação para representar o conhecido Nomograma de FROST \& SCHWALEN (1955), sendo considerado um modelo geral, pois é recomendado para diferentes tamanhos de bocais, pressões de operação e condições operacionais. No entanto, vale salientar que o mesmo modelo foi obtido a partir de condições ambientais médias do Arizona (Estados Unidos), as quais são distintas das condições ambientais brasileiras, dificultando a importação desse modelo para as condições do Brasil. 

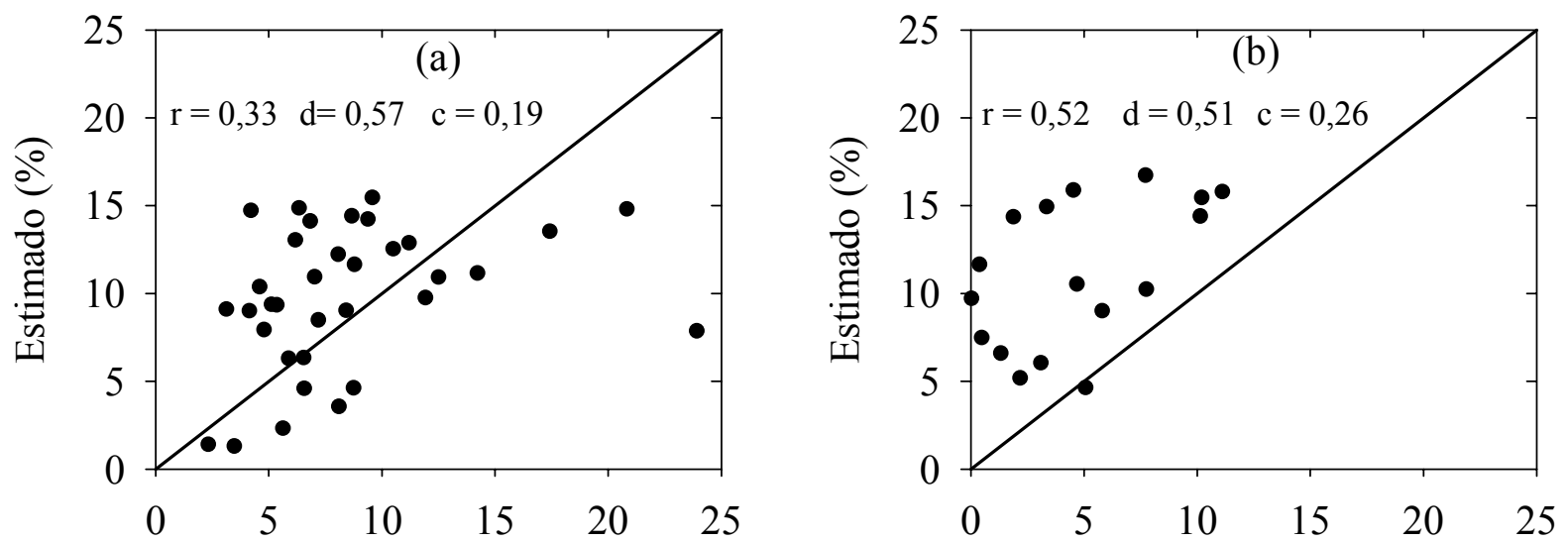

Observado (\%)
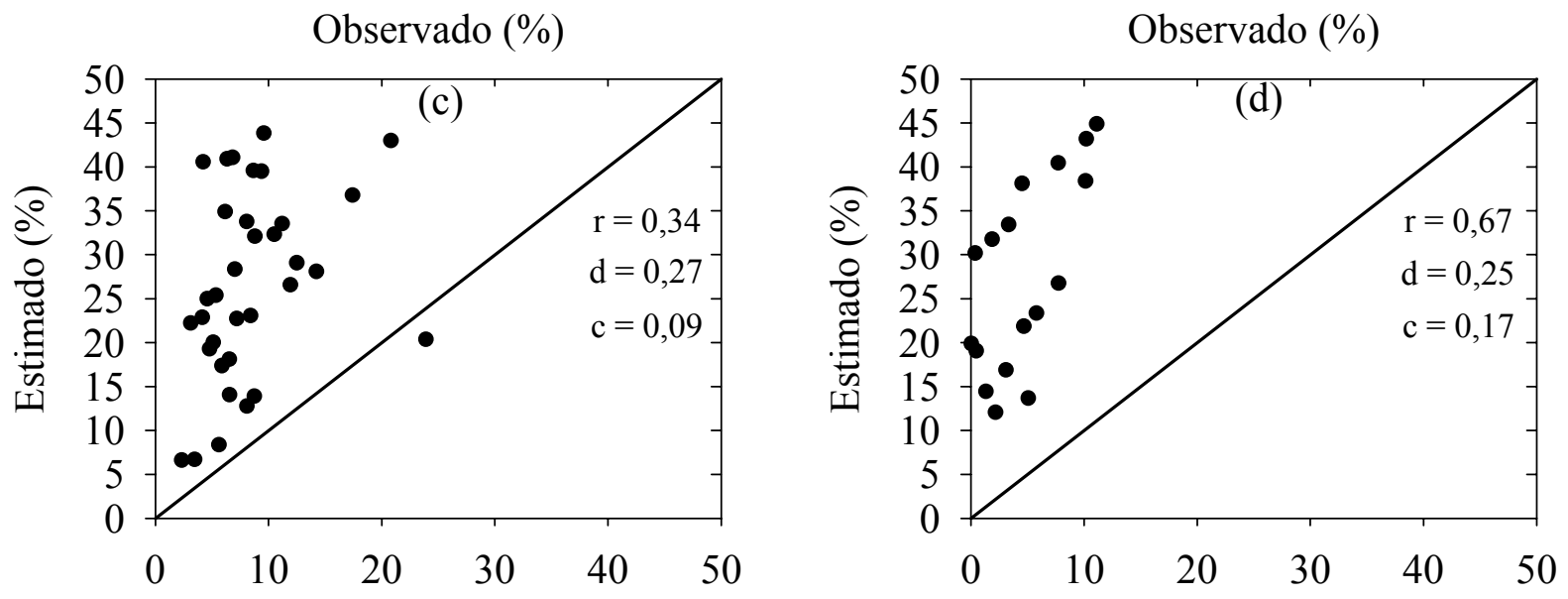

Observado (\%)
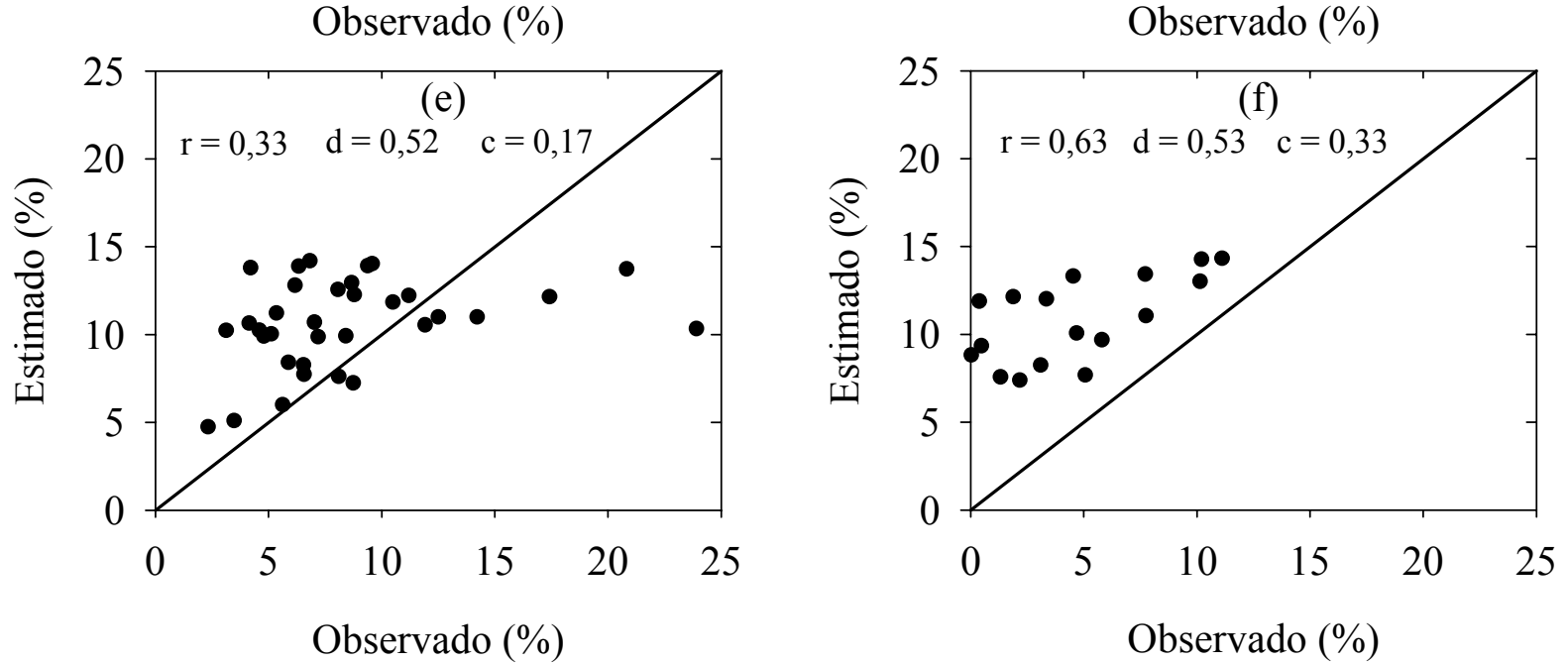

FIGURA 3. Valores de perdas de água por evaporação e arraste observados em testes de campo, respectivamente, com um único aspersor e com linhas laterais de aspersores, versus valores simulados pelos modelos: (a, b) PLAYÁN et al. (2005); (c, d) SEGINER et al. (1991), e (e, f) TARJUELO et al. (2000). Evaporation and wind drift loss values obtained from outdoor trials, for single-sprinkler and block irrigation set-up, respectively, versus simulated values according with the following models: (a, b) PLAYÁN et al. (2005); (c, d) SEGINER et al. (1991); and (e, f) TARJUELO et al. (2000). 
Além disso, mesmo tendo sido realizado um número considerado de ensaios de campo (700), esse não é um número grande se considerada a diversidade de combinações possíveis de ocorrer entre configurações de aspersores e condições ambientais, inviabilizando a utilização de um modelo geral aplicado à estimativa de perdas de água por evaporação e arraste em sistemas de aspersão convencional. Em trabalho realizado por FACI \& BERCERO (1991) e PLAYÁN et al. (2005), esses autores também encontraram perdas de água superiores ao estimado por este modelo, indicando que o modelo de TRIMMER (1987) tende a subestimar os valores de perdas de água por evaporação e arraste.

Avaliando o modelo de YAZAR (1984), esse apresentou desempenho "Péssimo" $(\mathrm{c}=0,09)$ em relação aos dados obtidos em testes de campo com um único aspersor (Figura 4c), gerando perdas menores do que realmente aconteceu. Já quando se utilizou o mesmo modelo para prever perdas de água por evaporação e arraste nos testes com linhas laterais de aspersores, esse modelo apresentou melhor ajuste (Figura 4d), com índice "c" de 0,53 (Sofrível). Isso pode ser atribuído ao fato de esse pesquisador ter realizado testes com linhas laterais de aspersores, usando bocais de tamanho $5,2 \mathrm{~mm}$ e $5,6 \mathrm{~mm}$, um pouco maiores que os usados neste trabalho e operando em intervalo de pressões semelhante. No caso desse modelo, já foram indicadas as limitações do mesmo, como, por exemplo, os diâmetros de bocais e intervalos de pressões de operação para qual o modelo tem validade.

Em razão de os modelos de simulação utilizados não terem apresentado boa adequação aos dados obtidos em testes de campo, nas condições locais de Lavras - MG, houve a necessidade de desenvolver modelos específicos para o aspersor em questão: um relacionado aos testes de campo com um único aspersor, e outro para ensaios com linhas laterais de aspersores, representados, respectivamente, pelas eqs. (9) e (10):

$$
\begin{aligned}
& \mathrm{EA}=0,00259 \mathrm{P}_{\mathrm{S}}+7,2781 \Delta \mathrm{e}^{0,5}+0,1252 \mathrm{U} \\
& \mathrm{EA}=-0,0177 \mathrm{P}_{\mathrm{S}}+4,2768 \Delta \mathrm{e}^{0,5}+3,7499 \mathrm{U}
\end{aligned}
$$

Os modelos gerados neste trabalho foram obtidos de acordo com o proposto por ORTEGA et al. (2000), TARJUELO (1999) e TARJUELO et al. (2000), e suas análises de adequações podem ser visualizadas pelas Figuras 4(e) e 4(f). O modelo desenvolvido para predizer perdas de água por evaporação e arraste para um único aspersor operando [eq.(9)] teve índice "c" $(0,18)$, melhor do que os apresentados pelos outros modelos usados neste trabalho; entretanto, ainda se enquadra na categoria "Péssimo", indicando que a formulação sugerida pelos autores recém-citados não é adequada para testes com um único aspersor NY-7. Por outro lado, o modelo para linhas laterais de aspersores [eq.(10)] apresentou índice "c" de 0,79, que se enquadra na categoria "Muito Bom", sendo indicada sua utilização. Entretanto, cabe salientar que os resultados observados se aplicam para configurações de aspersores semelhantes ao usado neste trabalho (bocais de 4,6 $\mathrm{mm} \mathrm{x}$ 4,0 mm), bem como intervalo de pressão entre $245 \mathrm{kPa}$ e $343 \mathrm{kPa}$.

Além da análise visual, por meio dos gráficos apresentados (Figura 3 e Figura 4), a Tabela 1 possibilita verificar a raiz do erro quadrado médio (REQM) que cada modelo de simulação apresentou e, dessa forma, pôde-se concluir que os dois modelos gerados neste trabalho se mostraram mais eficazes ao simular as perdas de água por evaporação e arraste para a configuração de aspersor em questão, nas condições locais brasileiras. 

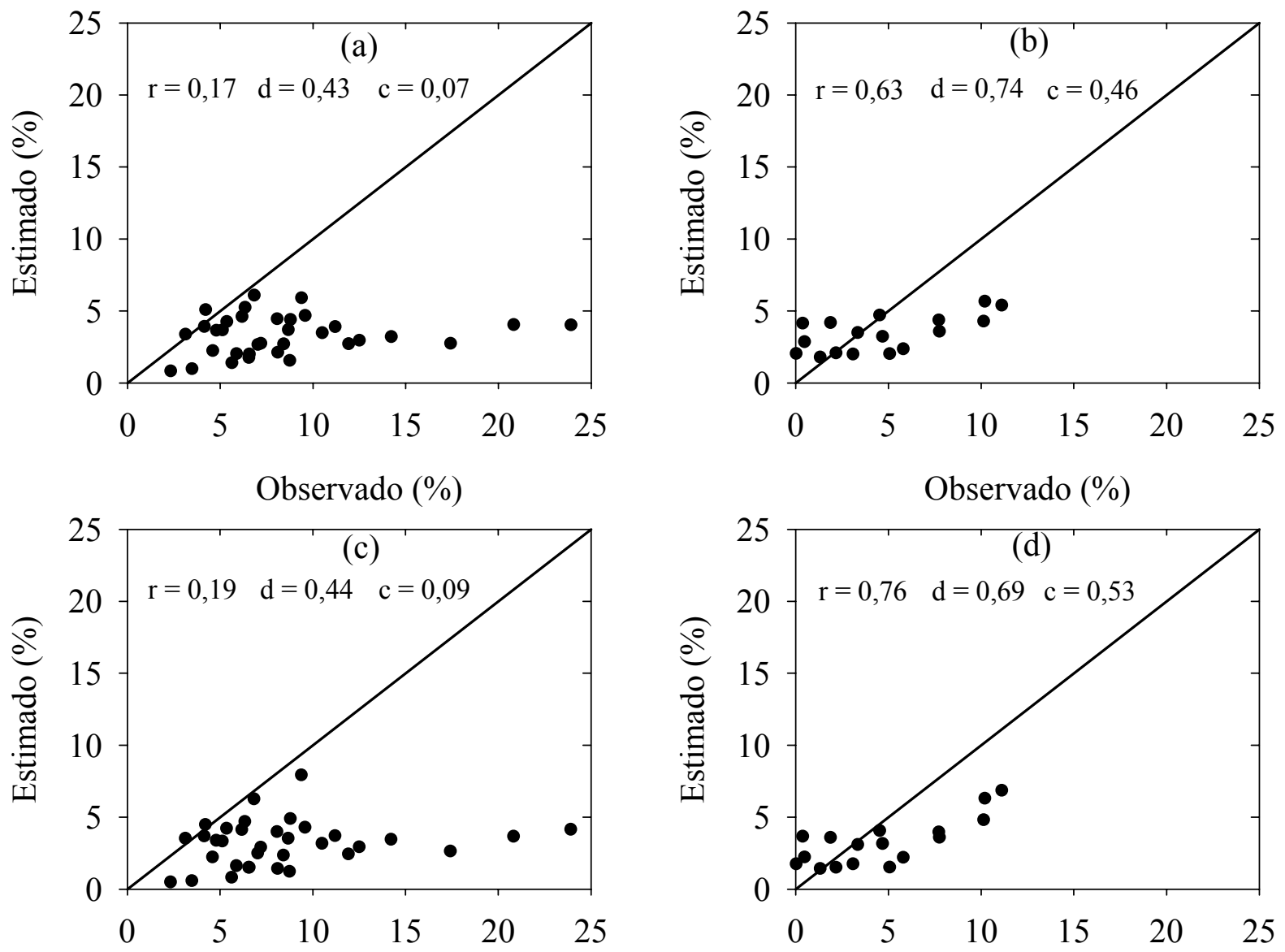

Observado $(\%)$
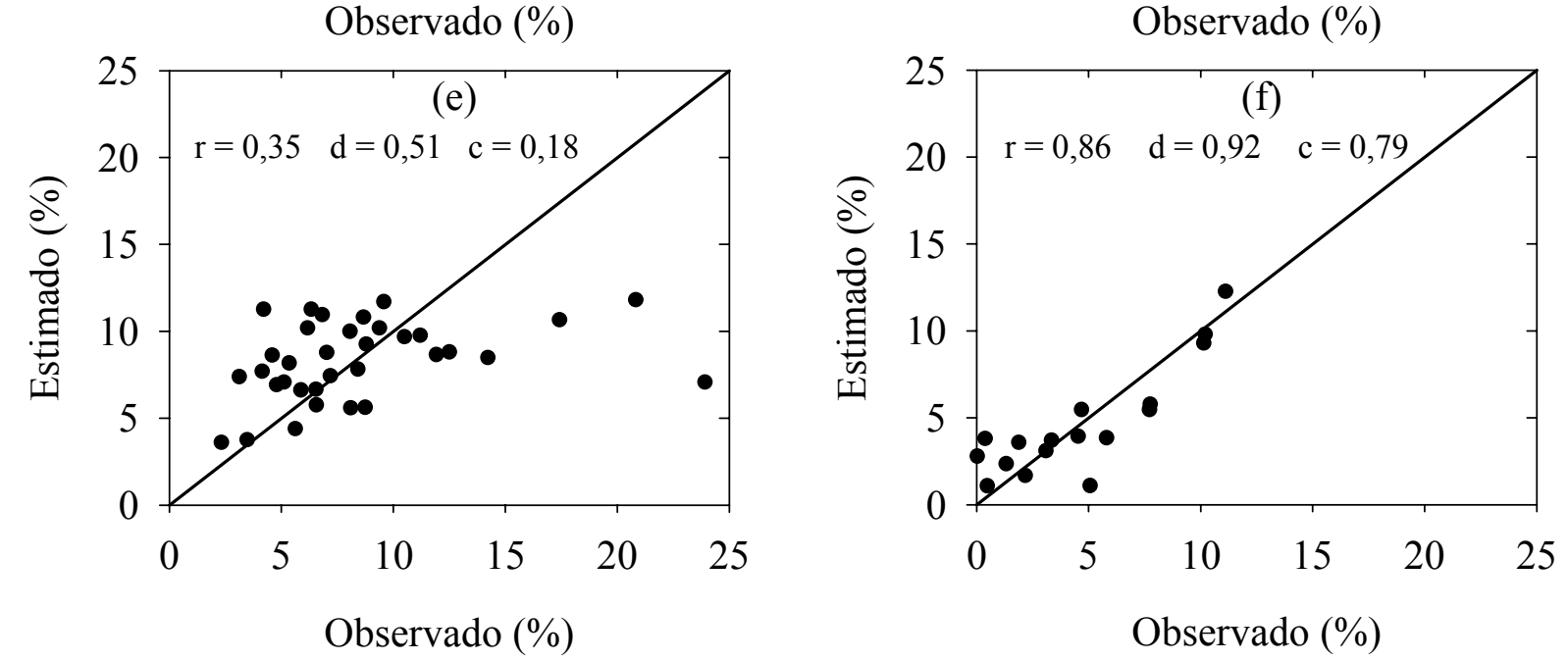

FIGURA 4. Valores de perdas de água por evaporação e arraste observados em testes de campo, respectivamente, com um único aspersor e com linhas laterais de aspersores, versus valores simulados pelos modelos: (a, b) TRIMMER (1987); (c, d) YAZAR (1984), e (e, f) propostos neste trabalho. Evaporation and wind drift loss values obtained from outdoor trials, for single-sprinkler and block irrigation set-up, respectively, versus simulated values according with the following models: (a, b) TRIMMER (1987); (c, d) YAZAR (1984); and (e, f) proposed equations. 
TABELA 1. Raiz do erro quadrado médio das perdas de água por evaporação e arraste (\%), segundo os diferentes modelos empíricos utilizados e também segundo os modelos propostos neste trabalho. Root mean square error (\%) of the evaporation and wind drift loss values obtained from the different empirical models used and proposed equations.

\begin{tabular}{|c|c|c|c|}
\hline \multirow{2}{*}{$\mathrm{N}^{\mathrm{o}}$} & \multirow{2}{*}{ Modelo } & \multicolumn{2}{|c|}{ REQM (\%) } \\
\hline & & Testes com um Único Aspersor & Testes com Laterais de Aspersores \\
\hline & ÁN et al. (2005) & 5,27 & 7,41 \\
\hline & JER et al. (1991) & 20,70 & 24,43 \\
\hline & JELO et al. (2000) & 5,07 & 6,72 \\
\hline & MER (1987) & 6,95 & 3,16 \\
\hline 5 I & $\mathrm{R}(1984)$ & 7,06 & 2,90 \\
\hline $6 \mathrm{~F}$ & & 4,47 & 1,81 \\
\hline
\end{tabular}

\section{CONCLUSÕES}

Os cinco modelos usados para realizar a simulação apresentaram desempenhos "Péssimo", "Mau" e, no máximo, "Sofrível".

É necessário muito cuidado na adoção de um dado modelo empírico para a estimativa das perdas de água por evaporação e arraste na irrigação por aspersão convencional, sendo importante analisar as limitações do modelo, como, por exemplo, diâmetro de bocal, pressão de operação e inclinação do jato, utilizadas para gerar o mesmo.

Nas condições locais brasileiras, os dois modelos gerados neste trabalho mostraram melhor adequação aos dados observados.

\section{REFERÊNCIAS}

BESKOW, S. Avaliação de modelos empíricos para estimativa das perdas de água por evaporação e arraste em sistemas de aspersão convencional. 2006. 94 f. Dissertação (Mestrado em Irrigação e Drenagem) - Universidade Federal de Lavras, Lavras, 2006.

CAMARGO, A.P.; SENTELHAS, P.C. Avaliação do desempenho de diferentes métodos de estimativa da evapotranspiração potencial no Estado de São Paulo, Brasil. Revista Brasileira de Agrometeorologia, Santa Maria, v.5, n.1, p.89-97, 1997.

FACI, J.M.; BERCERO, A. Efecto del viento en la uniformidad y en las perdidas por evaporación y arraste en riego por aspersión. Investigation Agrária - Produccion y Proteccion Vegetable, Madrid, v.6, n.2, p.171-80, 1991.

FACI, J.M.; SALVADOR, R.; PLAYÁN, E.; SOURELL, H. Comparison of fixed and rotating spray plate sprinklers. Journal of Irrigation and Drainage Engineering, Reston, v.127, n.4, p.22433, 2001.

FROST, K.R.; SCHWALEN, H.C. Sprinkler evaporation losses. Agricultural Engineering, St. Joseph, v.36, n.8, p.526-8, 1955.

KELLER, J.; BLIESNER, R.D. Sprinkle and trickle irrigation. New York: AnaviBook - Van Nostrand Reinhold, 1990. 652 p.

KINCAID, D.C. Spraydrop kinetic energy from irrigation sprinklers. Transactions of the ASAE, St. Joseph, v.39, n.3, p.847-53, 1996.

KINCAID, D.C.; SOLOMON, K.H.; OLIPHANT, J.C. Drop size distributions for irrigation sprinklers. Transactions of the ASAE, St. Joseph, v.39, n.3, p.839-45, 1996. 
KOHL, K.D.; KOHL, R.A.; DeBoer, D.W. Measurement of low pressure sprinkler evaporation loss. Transactions of the ASAE, St. Joseph, v.30, n.4, p.1.071-4, 1987.

MONTERO, J.; ORTEGA, J.F.; TARJUELO, J.M.; HONRUBIA, F.T. Análisis de la perdidas por evaporación y arraste en el riego por aspersión. In: CONGRESSO NACIONAL DE RIEGOS, 15., 1997, Madrid. Anais... Madrid: Asociación Española de Riegos y Drenajes, 1997. p.144-52.

ORTEGA, J.F.; TARJUELO, J.M.; MONTERO, J.; DE JUAN, J.A. Discharge efficiency in sprinkling irrigation: analysis of the evaporation and drift losses in semi-arid areas. Agricultural Engineering International, the CIGR Ejournal, v.2, p.1-21, 2000. Disponível em: $<$ http://cigrejournal.tamu.edu>. Acesso em: 28 jun. 2006.

PLAYÁN, E.; SALVADOR, R.; FACI, J.M.; ZAPATA, N.; MARTINEZ-COB, A.; SÁNCHEZ, I. Day and night wind drift and evaporation losses in sprinkler solid-sets and moving laterals. Agricultural Water Management, Amsterdam, v.76, n.3, p.139-59, 2005.

SEGINER, I.; KANTZ, D.; NIR, D. The distortion by wind of the distribution patterns of single sprinklers. Agricultural Water Management, Amsterdam, v.19, n.4, p.341-59, 1991.

SMAJSTRLA, A.G.; ZAZUETA, F.S. Evaporation loss during sprinkler irrigation. Gainesville: Agricultural and Biological Engineering Department, 2003. 8 p.

TARJUELO, J.M. El riego por aspersión y su tecnologia. 2.ed. Madri: Mundi-Prensa, 1999. 565 p.

TARJUELO, J.M.; ORTEGA, J.F.; MONTERO, J.; JUAN, J.A. Modelling evaporation and drift losses in irrigation with medium size impact sprinklers under semi-arid conditions. Agricultural Water Management, Amsterdam, v.43, n.3, p.263-84, 2000.

THOMPSON, A.L.; GILLEY, J.R.; NORMAN, J.M. A sprinkler water droplet evaporation and plant canopy model: I. Model Development. Transactions of the ASAE, St. Joseph, v.36, n.3, p.735-41, maio/jun. 1993.

TRIMMER, W.L. Sprinkler evaporation loss equation. Journal of Irrigation and Drainage Engineering, New York, v.113, n.4, p.616-20, 1987.

YAZAR, A. Evaporation and drift losses from sprinkler irrigation systems under various operating condition. Agricultural Water Management, Amsterdam, v.8, n.4, p.439-49, 1984. 